Case Reports
in Dermatology

\title{
Vitiligo with Progressive Repigmentation during Secukinumab Treatment in a Patient with Psoriatic Arthritis: A Case Report
}

\author{
Domenico Giordano $^{a} \quad$ Francesca Magri $^{a} \quad$ Flavia Persechino $^{b}$ \\ Alejandra Lepore ${ }^{c}$ Romina Verde ${ }^{d}$ Alessandro Capalbo ${ }^{a}$ \\ Severino Persechino ${ }^{a}$ \\ aNESMOS Department, Dermatology Unit, Sant'Andrea Hospital Faculty of Medicine and \\ Psychology, Sapienza University of Rome, Rome, Italy; ${ }^{b}$ Department of Clinical and \\ Molecular Medicine, Sapienza University of Rome, Rome, Italy; ' Department of \\ Dermatology, Hospital Héroes de Malvinas, Buenos Aires, Argentina; dDepartment of \\ Clinical Dermatology, Sant'Andrea Hospital, Rome, Italy
}

\section{Keywords}

Psoriatic arthritis $\cdot$ Repigmentation · Secukinumab · Vitiligo

\begin{abstract}
The proposed role of interleukin (IL)-17 in vitiligo pathogenesis, as well as the possible action of anti-IL-17A drugs on vitiligo, are not fully understood. The appearance of vitiligo as a paradoxical effect of treatment with anti-tumor necrosis factor- $\alpha$ drugs is an event well known in the literature, but is rarely described with anti-IL-17A drugs. In this case report, we describe a 42-year-old woman who developed new-onset vitiligo with repigmentation during successful secukinumab therapy for psoriatic arthritis. After 1 year of secukinumab therapy, vitiligo affecting $>85 \%$ of the skin was evident on clinical and dermatoscopic examination, together with small, repigmented lesions. In depigmented lesions, reflectance confocal microscopy (RCM) showed disappearance of the bright dermal papillary rings normally seen at the dermo-epidermal junction. In repigmented lesions, activated dendritic melanocytes were observed on $\mathrm{RCM}$. The patient continued to receive secukinumab, and continued to experience a slow and
\end{abstract}




\section{Case Reports in Dermatology}

Case Rep Dermatol 2021;13:209-215

DOI: $10.1159 / 000510831$

(c) 2021 The Author(s). Published by S. Karger AG, Basel www.karger.com/cde

Giordano et al.: Vitiligo with Progressive Repigmentation during Secukinumab Therapy

progressive repigmentation. Our case shows that anti-IL-17A biological agents for chronic inflammatory diseases may be associated with the development of new-onset vitiligo that improves over time with ongoing therapy. Therefore, physicians should be aware of the possibility of this rare paradoxical skin reaction in patients receiving secukinumab, and that it may not be necessary to discontinue secukinumab to achieve repigmentation.

(C) 2021 The Author(s)

Published by S. Karger AG, Basel

\section{Introduction}

The pathogenesis of the skin hypopigmentation disorder vitiligo is not fully understood, and treatment options are limited [1]. A positive correlation between serum interleukin (IL)17 levels and the extent and duration of vitiligo has been reported, and marked decreases in IL-17 levels have been observed after successful treatment of vitiligo with narrowband ultraviolet B therapy [2, 3]. It has therefore been suggested that IL-17 may play an important role in vitiligo pathogenesis, and that IL-17 inhibition may be a promising therapy $[1,4]$.

However, a case of new-onset vitiligo was reported in a patient receiving the anti-IL-17A biological agent secukinumab [5], and thus the direct pathological role of IL-17 in vitiligo has been called into question [1]. In addition, a more recent case report described the development of vitiligo in a patient receiving adalimumab, who, after discontinuation of adalimumab and initiation of secukinumab, showed gradual resolution of vitiligo and other psoriatic symptoms [6]. In this case, it was hypothesized that the negative modulation of IL-17 determined the improvement of both psoriasis and vitiligo [6].

Here, we describe the case of a patient with psoriatic arthritis (PsA) who developed newonset vitiligo and repigmentation during ongoing secukinumab therapy that was examined by reflectance confocal microscopy (RCM).

\section{Case Presentation}

Our patient was a 42-year-old Caucasian woman who was diagnosed with psoriasis at 16 years of age. She had initially received topical corticosteroids without response, and then systemic ciclosporin $200 \mathrm{mg}$, with a good outcome for 2 years followed by progressive loss of response. She developed severe bilateral hand and wrist joint symptomatology with dactylitic digits, and ultrasound examination revealed tenosynovitis and joint effusion. Extensive skin involvement was observed, with a Psoriasis Area Severity Index (PASI) score of 23.7. The patient was diagnosed with PsA. Ciclosporin was discontinued and she received methotrexate for 4 months without improvement, after which she was switched to secukinumab (300 $\mathrm{mg} / \mathrm{month}$ ). Six months later, the patient achieved a 75\% reduction in PASI score and reported a score of 2 on a 0 - to $10-\mathrm{cm}$ visual analogue scale of joint and pain stiffness. At 9 months, she had clinical resolution of psoriasis (PASI score of 0 ) and disappearance of the articular manifestations of PsA (visual analogue scale score of 0 ).

Vitiligo appeared after 1 year of secukinumab therapy. The patient had no personal or family history of vitiligo. Clinical and dermatoscopic examination revealed vitiligo covering $>85 \%$ of the skin, with small areas of repigmentation (Fig. 1). Examination by RCM (VivaScope 1500; Maving $\mathrm{GmbH}$, Munich, Germany) revealed disappearance of the bright dermal

\section{Karger'=}




\section{Case Reports in Dermatology}

Case Rep Dermatol 2021;13:209-215

DOI: $10.1159 / 000510831$

C 2021 The Author(s). Published by S. Karger AG, Basel www.karger.com/cde

Giordano et al:: Vitiligo with Progressive Repigmentation during Secukinumab Therapy

papillary rings normally seen at the dermo-epidermal junction in depigmented vitiligo lesions (Fig. 2) and activated dendritic melanocytes in repigmented lesions (Fig. 3). The patient continued to receive secukinumab, during which she experienced progressive repigmentation over the next 16 months of follow-up.

\section{Discussion}

Our case adds to the evidence that - although very rare, with only 1 other case reported to our knowledge [5] - new-onset vitiligo can occur during therapy with anti-IL-17A biological agents for chronic inflammatory diseases. Most reports of new-onset vitiligo occurring during biological therapy have been on patients receiving anti-tumor necrosis factor- $\alpha$ (TNF- $\alpha$ ) agents $[5,7]$. In a case series of 18 patients with new-onset vitiligo during biological therapy, 14 patients $(77.8 \%)$ were receiving anti-TNF- $\alpha$ agents, 3 patients were receiving an anti-IL$12 / 23$ agent (16.7\%), and 1 patient (5.6\%) was receiving secukinumab [5]. The average duration of exposure before the onset of vitiligo ranged from 13.9 to 16.5 months [5], which is consistent with vitiligo developing after 12 months of secukinumab therapy in our patient. In the case series, biological therapy was continued with stabilization of vitiligo or partial repigmentation in 12 of the 18 patients (66.6\%) [5], and similarly, progressive repigmentation of the vitiligo lesions was also observed in our patient during ongoing secukinumab therapy. Collectively, these observations indicate that a favorable outcome is possible without discontinuation of biological therapy in patients with new-onset vitiligo.

Our patient's vitiligo lesions were examined using RCM, which is a noninvasive diagnostic tool providing real-time images that approach cellular histological resolution. Use of RCM to diagnose and monitor inflammatory skin disease represents a relatively new field of interest [8-10]. Compared with the surrounding skin structures, melanin has a high reflectance index, allowing melanocytes, which are progressively lost in vitiligo lesions, to be clearly visualized with RCM $[9,10]$. As demonstrated in our case and in previous studies, RCM is an important instrument for the evaluation of vitiligo and repigmentation [8-10]. The absence of bright dermal papillary rings at the dermo-epidermal junction level is indicative of vitiligo, and the presence of dendritic melanocytes is a sign of repigmentation $[9,10]$, both of which were observed in our patient.

It is possible that new-onset vitiligo in patients receiving biological agents for inflammatory diseases is related to the underlying disease, especially when the inflammatory disease is poorly controlled [5]. However, our patient had a long history of psoriasis with no vitiligo, and her PsA was well controlled with secukinumab. Furthermore, only 3 of the 18 patients in the aforementioned case series had to switch biological agents because of uncontrolled inflammatory disease [5]. Therefore, it has been suggested that vitiligo may be a paradoxical skin reaction to biological agents, and that physicians should be aware of the possibility of new-onset vitiligo during biological therapy, particularly with anti-TNF- $\alpha$ agents $[5,7]$.

Complex mechanisms, probably involving multiple proinflammatory and anti-inflammatory cytokine pathways, are responsible for the destruction of melanocytes and the onset of vitiligo associated with biological therapy. Presumably, anti-TNF- $\alpha$ drugs, which act relatively upstream in the inflammatory cascade, trigger a wider range of anti- and proinflammatory cytokine changes compared with secukinumab, which acts on downstream anti-IL-17 signaling [11]. This may explain why anti-TNF- $\alpha$ drugs pose a greater risk of vitiligo than other biological drugs.

\section{Karger'=}




\section{Case Reports in Dermatology}

Giordano et al.: Vitiligo with Progressive Repigmentation during Secukinumab Therapy

Until recently, most information regarding the efficacy of anti-IL-17 drugs in patients with pre-existing vitiligo was derived from case reports of patients with vitiligo as a comorbidity of the underlying inflammatory disease for which these drugs are indicated. For example, secukinumab was reported to result in an improvement in all conditions in a patient with plaque psoriasis and concomitant vitiligo and lichen striatus [12]. However, in a more recent pilot study of secukinumab for the treatment of active vitiligo, 7 of 8 patients (87.5\%) showed progression of vitiligo with no clear signs of repigmentation [1]. This is consistent with the observation that the prognosis tends to be worse in patients with pre-existing vitiligo who are treated with biological agents for chronic inflammatory diseases than in those with new-onset vitiligo [5].

In conclusion, new-onset vitiligo may develop during therapy with secukinumab for chronic inflammatory diseases, but may improve over time if treatment is continued. The treating physician therefore should be aware of the possibility of such a paradoxical adverse cutaneous reaction and of how to proceed should vitiligo develop. A clinical diagnosis of vitiligo should be confirmed by RCM, and an otherwise successful biological treatment strategy should not be discontinued unless the impact of vitiligo on quality of life outweighs the benefits of biological therapy for the underlying inflammatory disease.

\section{Acknowledgments}

The authors would like to thank Sarah Greig, PhD, of Springer Healthcare Communications, who edited and styled the manuscript for submission. This medical writing support was funded by Novartis Farma, Italy.

\section{Statement of Ethics}

The patient has provided written informed consent for publication including photographs. The research was conducted ethically in accordance with the World Medical Association Declaration of Helsinki.

\section{Conflict of Interest Statement}

The authors have no conflicts of interest to declare.

\section{Funding Sources}

No funding was received.

\section{Author Contributions}

D. Giordano: clinical evaluation of the patient, main author of the manuscript, collection of written informed patient consent, and clinical photographs. F. Magri: bibliographic research

\section{Karger'=}




\section{Case Reports in Dermatology}

\begin{tabular}{l|l}
\hline DOI: 10.1159/000510831 & $\begin{array}{l}\text { (c) } 2021 \text { The Author(s). Published by S. Karger AG, Basel } \\
\text { www.karger.com/cde }\end{array}$
\end{tabular}

Giordano et al.: Vitiligo with Progressive Repigmentation during Secukinumab Therapy

and revision of grammatical and lexical terms. F. Persechino: RCM analysis, writing of the part of the manuscript related to RCM, taking of RCM images, and writing of the related captions. A. Lepore: bibliographic research and review of the manuscript's contents. R. Verde: bibliographic research and revision of grammatical and lexical terms. A. Capalbo: bibliographic research and co-participation in the clinical evaluation and follow-up of the patient. S. Persechino: supervision of the entire clinical case and review of the manuscript's contents. All authors agree to be accountable for all aspects of the work in ensuring that questions related to the accuracy or integrity of any part of the work are appropriately investigated and resolved.

\section{References}

1 Speeckaert R, Mylle S, van Geel N. IL-17A is not a treatment target in progressive vitiligo. Pigment Cell Melanoma Res. 2019 Nov;32(6):842-7.

2 Bassiouny DA, Shaker O. Role of interleukin-17 in the pathogenesis of vitiligo. Clin Exp Dermatol. 2011 Apr;36(3):292-7.

3 Hegazy RA, Fawzy MM, Gawdat HI, Samir N, Rashed LA. T helper 17 and Tregs: a novel proposed mechanism for NB-UVB in vitiligo. Exp Dermatol. 2014 Apr;23(4):283-6.

4 Singh RK, Lee KM, Vujkovic-Cvijin I, Ucmak D, Farahnik B, Abrouk M, et al. The role of IL-17 in vitiligo: a review. Autoimmun Rev. 2016 Apr;15(4):397-404.

5 Méry-Bossard L, Bagny K, Chaby G, Khemis A, Maccari F, Marotte H, et al. New-onset vitiligo and progression of pre-existing vitiligo during treatment with biological agents in chronic inflammatory diseases. J Eur Acad Dermatol Venereol. 2017 Jan;31(1):181-6.

6 Palazzo G. Resolution of post-adalimumab vitiligo with secukinumab in a patient with psoriasis vulgaris. Oxf Med Case Rep. 2020 Jan;2020(1):omz134.

7 Bae JM, Kim M, Lee HH, Kim KJ, Shin H, Ju HJ, et al. Increased risk of vitiligo following anti-tumor necrosis factor therapy: a 10-year population-based cohort study. J Invest Dermatol. 2018 Apr;138(4):768-74.

8 Ardigo M, Malizewsky I, Dell'anna ML, Berardesca E, Picardo M. Preliminary evaluation of vitiligo using in vivo reflectance confocal microscopy. J Eur Acad Dermatol Venereol. 2007 Nov;21(10):1344-50.

9 Lai LG, Xu AE. In vivo reflectance confocal microscopy imaging of vitiligo, nevus depigmentosus and nevus anemicus. Skin Res Technol. 2011 Nov;17(4):404-10.

10 Li W, Wang S, Xu AE. Role of in vivo reflectance confocal microscopy in determining stability in vitiligo: a preliminary study. Indian J Dermatol. 2013 Nov;58(6):429-32.

11 Speeckaert R, Lambert J, Grine L, Van Gele M, De Schepper S, van Geel N. The many faces of interleukin-17 in inflammatory skin diseases. Br J Dermatol. 2016 Nov;175(5):892-901.

12 Yang CW, Tsai TF. Image Gallery: resolution of lichen striatus in a patient with coexisting chronic plaque psoriasis and vitiligo during secukinumab treatment. Br J Dermatol. 2017 May;176(5):e96. 


\section{Case Reports in Dermatology}
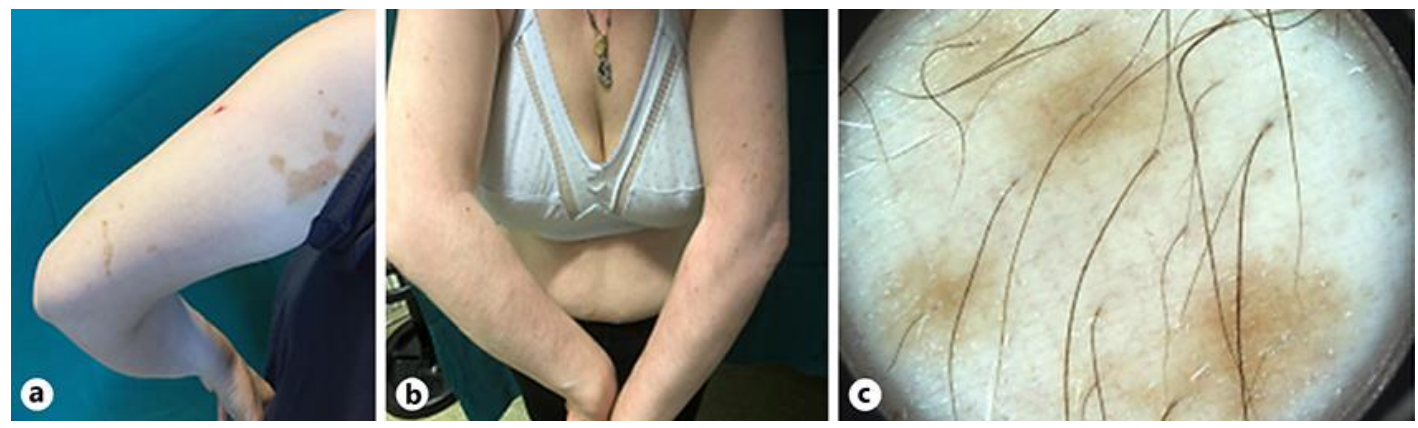

Fig. 1. Photographic images of vitiligo with isolated areas of pigmentation on the upper limbs (a) and trunk (b), highlighted by dermatoscopic examination (c).
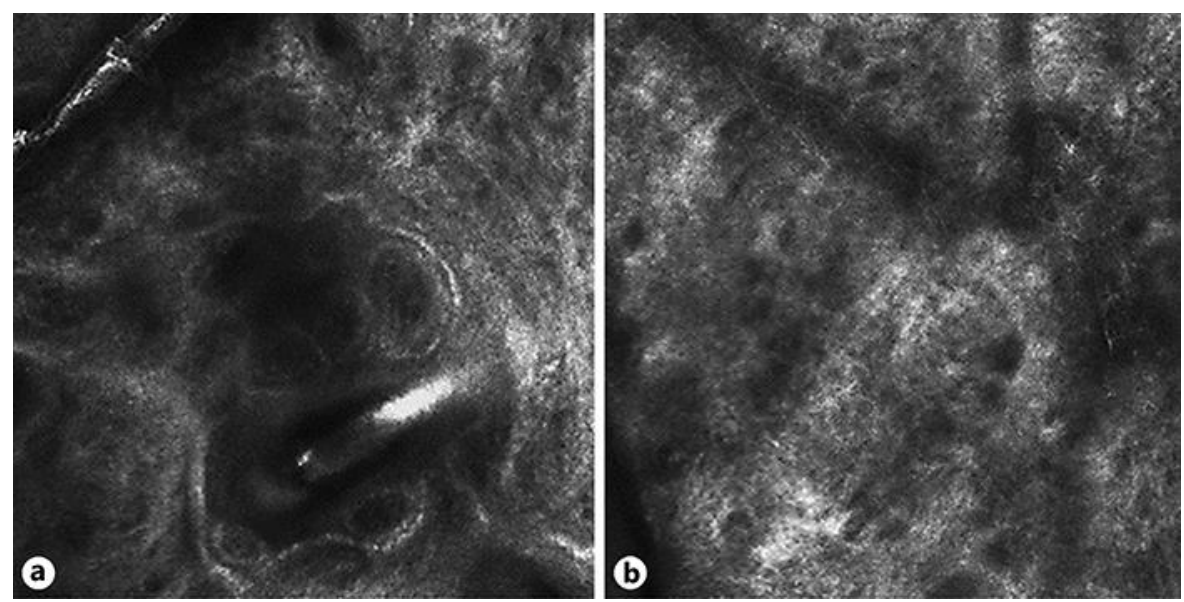

Fig. 2. Reflectance confocal microscopy images of apparently normal skin with low pigmentation in the dermal layer (a) and vitiligo lesions (b), showing disappearance of the normal ring structures at the dermoepidermal junction level with only a shadow of the pre-existing dermal papillary rings and no bright structures detected in the upper epidermal layers. 


\section{Case Reports in Dermatology}
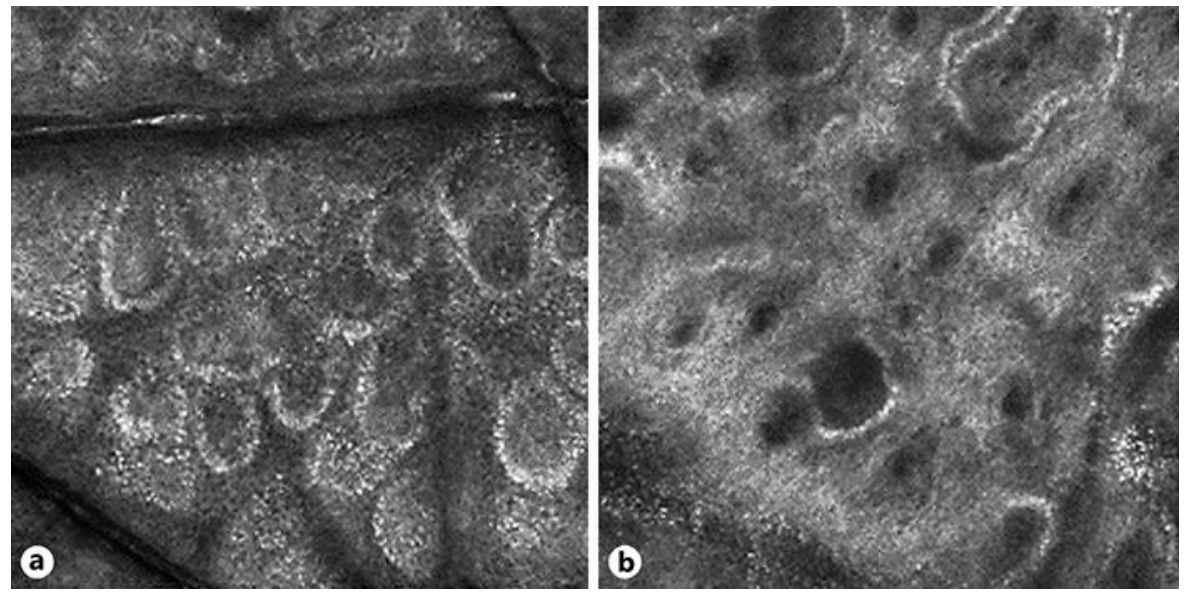

Fig. 3. Reflectance confocal microscopy images of repigmented areas containing melanocytes and large, bright pigmented keratinocytes (a) and apparently normal skin with low pigmentation in the epidermal layer (b). 